\title{
Musical instrument recordings made with a fiber Fabry-Perot cavity: photonic guitar pickup
}

\author{
Nicholas Ballard, ${ }^{1}$ Daniel Paz-Soldan, ${ }^{1}$ Peter Kung, ${ }^{2}$ and Hans-Peter Loock ${ }^{1, *}$ \\ 'Department of Chemistry, Queen's University, Kingston, Ontario, K7L 3N6, Canada \\ ${ }^{2}$ QPS Photronics, 217 St.Louis Ave Pointe Claire, Montreal, Quebec, H9R 5L7 Canada \\ ${ }^{*}$ Corresponding author: hploock@chem.queensu.ca
}

Received 21 January 2010; revised 4 March 2010; accepted 8 March 2010; posted 9 March 2010 (Doc. ID 123091); published 7 April 2010

\begin{abstract}
A $1 \mathrm{~cm}$ long, low-finesse fiber-optic cavity was used as a transducer for the vibrations of the soundboard of an acoustic guitar and of a violin. The reflected light is detected and then amplified and recorded using conventional audio instrumentation. The fiber-optic pickup is found to have a high response range in both amplitude (up to $100 \mu \mathrm{m}$ displacement) and audio frequency (DC to $20 \mathrm{kHz}$ ) and good linearity up to a displacement of $225 \mu \mathrm{m}$. The audio noise is found to arise from the fiber-optic cables and, to a lesser extent, from the laser and laser driver. (c) 2010 Optical Society of America

OCIS codes: $\quad 060.3735,120.7280,230.1040$.
\end{abstract}

\section{Introduction}

It is well known that the reflection spectra of fiber Bragg gratings (FBGs) shift as the FBGs are strained or bent. Consequently, FBGs find increasing use as transducers for vibration or strain, aside from their more "conventional" uses as narrowband reflectors or bandstop filters.

In a previous article we described the use of single FBGs as "pickups" for an acoustic guitar [1]. FBGs have been used previously for the detection of ultrasound, e.g., in medical applications [2-4], underwater monitoring [ [5-7], and in photoacoustic spectroscopy $[8,9]$. With regard to their use as instrument pickups FBGs have a number of advantages over the commonly employed piezoelectric pickups, such as low mass, very large frequency response range, and immunity to electromagnetic interference. Sometimes these audio frequency interference, such as the " $60 \mathrm{~Hz}$ hum" generated by poorly grounded musical instruments, amplifiers, and other equipment, can only be removed by electronic filtering.

While monitoring the shift of the FBG reflection wavelength is adequate for the large-amplitude, low-

0003-6935/10/112198-06\$15.00/0

(C) 2010 Optical Society of America frequency motions that are relevant to structural health monitoring of bridges, buildings, and the like, other applications such as high-fidelity audio recordings require a higher sensitivity to low-amplitude vibrations, flat frequency response, a large dynamic range in vibration amplitude $(1 \mu \mathrm{m}$ to $200 \mu \mathrm{m}$ for an acoustic guitar), and a wide audio frequency response (DC to $22 \mathrm{kHz}$ ). When high sensitivity to low-amplitude vibration is required, fiber grating structures present a number of options, including very long FBGs, $\pi$-shifted FBGs and, of relevance to this study, Fabry-Perot (FP) cavities that are formed by two identical FBGs [Figs. 1(a) and 1(b)]. With such cavities a detection limit to static and dynamic strain at a level better than $1 \mathrm{p} \varepsilon$ has been demonstrated $[10,11]$. In some of the most sensitive systems the laser is actively locked to the FBG attenuation feature [12] or one of the FP cavity resonances $[10,13]$ and the strain on the FBG or cavity, i.e., its variation in length, is measured from the error signal used to track the resonance. A simpler, but less sensitive, scheme simply involves tuning a narrowband laser near the midattenuation point of a cavity fringe and measuring the transmitted or, preferably, reflected intensity as the cavity spectrum oscillates with vibration or strain. The latter method was implemented in this study. 
The FBG-FP cavity sensor head has some advantages over the single FBG that was employed as a strain sensor in the previous article [1]. When the single FBG is placed on the soundboard of an instrument it may show little sensitivity to those frequencies that form a nodal line through the FBG on the sound board. Since the sensitive region of the FBGFP cavity is distributed over a longer strand of fiber ( $10 \mathrm{~mm}$ in this case) there is less concern about missing frequencies. More importantly, the sensitivity and dynamic range of the strain amplitude are independently adjustable through the cavity parameters, i.e., free-spectral range (given by the cavity length) and finesse (FBG reflectivity). In the present study, we selected FBGs with a low reflectivity and placed them apart by a comparably large distance. The cavity parameters were chosen to match the guitar's vibration amplitude, here, up to about $100 \mu \mathrm{m}$.

\section{Experimental Setup}

The transducer system consists of a narrow linewidth distributed feedback laser, which is used to interrogate a low-finesse FBG-FP cavity, an optical circulator and a photodetector. Light reflected by the FBG-FP cavity is diverted to the photodetector by the circulator. The fiber cavity is mounted on an acoustic guitar using adhesive tape, and the vibration experienced by the guitar body upon playing the instrument changes the reflectance spectrum of the fiber cavity as described below. Three different fiber cavities (Vibrofiber, QPS Photronics, Montréal, QC) with two identical FBGs (length $300 \mu \mathrm{m}$, reflectivity $5-10 \%$ ) spaced at distances of $5 \mathrm{~mm}, 10 \mathrm{~mm}$, and $25 \mathrm{~mm}$ were used as acoustic vibration sensors. The FBG-FP cavities were different in their freespectral ranges and the widths of the cavity resonance modes. At constant FBG reflectivity the free-spectral range decreases with increasing cavity length, whereas the widths of the fringes decrease. Figure 1(c) shows the reflectance spectrum of the $10 \mathrm{~mm}$ cavity obtained by sweeping a widely tunable diode laser (Ando AQ4320D, $5 \mathrm{~mW}$ ) across the wavelength range of the cavity. The envelope is formed by the reflectance spectra of the FBGs, whereas the narrow fringes correspond to longitudinal cavity modes. The sensitivity of the strain measurement depends on the slope of the reflection spectrum near the midreflection point of a cavity mode and was found to be highest for the longest cavity, as expected. At the midreflection point the sensitivity of the measurement and the linear dynamic response range is largest. As the guitar is played, the strain on the FBGs themselves may shift their reflectance spectrum slightly [the envelope in Fig. 1(c)], but this effect can be neglected. More importantly, variation in the cavity length also shifts the wavelengths of the cavity resonances.

A compact and inexpensive high power distributed feedback laser (butterfly laser, AIFOtec, $>95 \mathrm{~mW} / \mathrm{A}$ ) centered at $1542.14 \mathrm{~nm}$ with a linewidth of $200 \mathrm{MHz}$ was used to interrogate the FBG-FP cavities. Using a
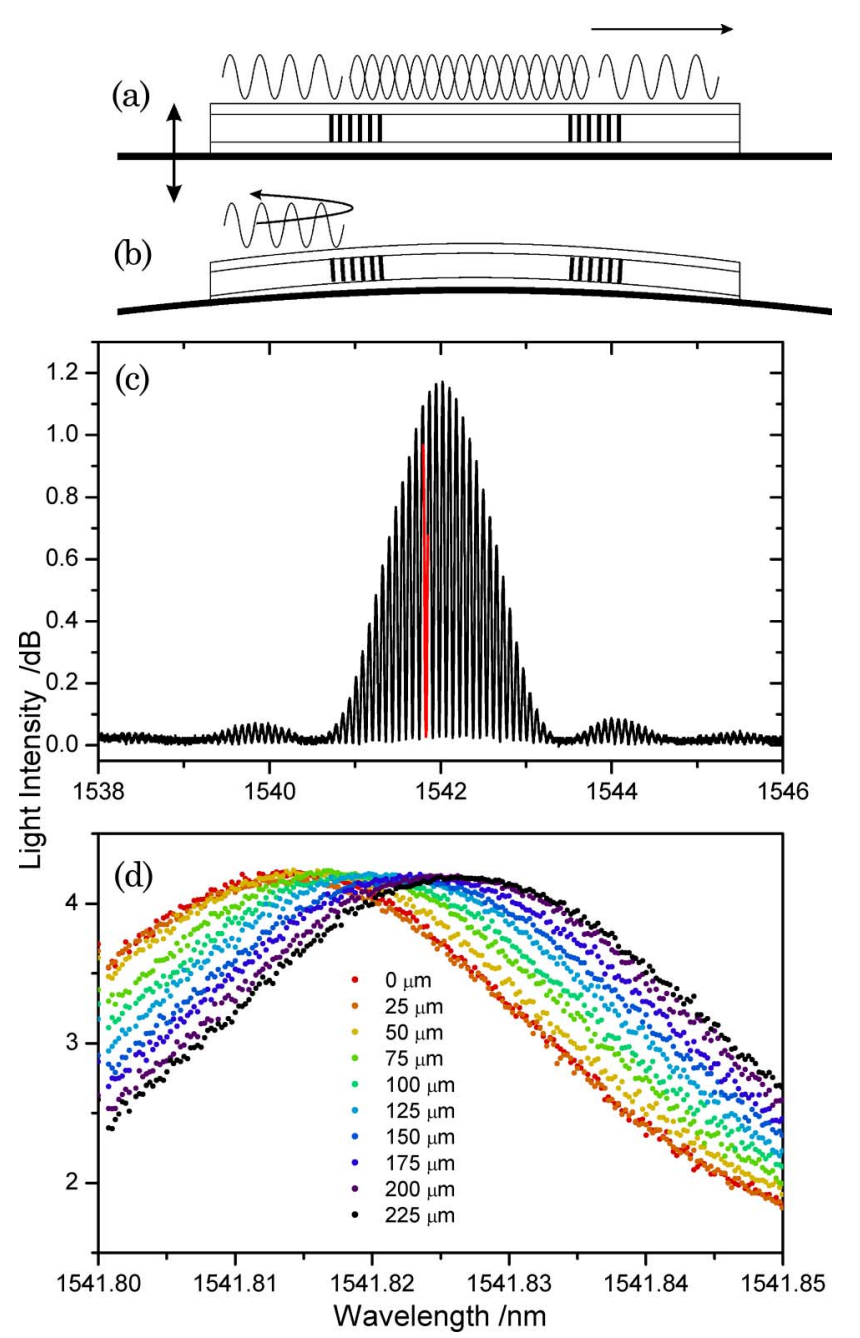

Fig. 1. (Color online) Fabry-Perot cavity made of two FBGs (a) in resonance and (b) not in resonance with incoming light. (c) Reflection spectrum of $10 \mathrm{~mm}$ long fiber cavity, (d) shift of one cavity fringe in the wavelength window indicated by the red line in (c) in response to the indentation of the sound board onto which the fiber cavity is affixed.

feedback algorithm the laser was periodically tuned to the midreflection point of one of the cavity fringes near the attenuation maxima of the two FBGs. The light reflected from the cavity was directed through an optical circulator (FDK Corp., YC-1100-155) to an InGaAs photodiode detector (Thorlabs, DET10C, $10 \mathrm{~ns}$ rise/fall time). The output current of the photodiode was dropped across a $50 \mathrm{k} \Omega$ terminating resistor (Thorlabs, VT1), and the resulting voltage was routed to a USB audio interface (Edirol, UA-25EX). The audio interface offers the first stage of gain for the electrical signal as well as a number of features for signal conditioning, but in all results shown below it was only used as an analog to digital converter whose output was sent to a computer via USB protocol.

The FBG-FP cavities were affixed to the soundboard of a hollow-body acoustic guitar (SC-MAH, Simon \& Patrick, Baie D'Urfé, QC) using adhesive tape (Fig. 2). Recordings were compared from cavities 
placed in different locations on the guitar, and it was determined that the most desirable position for the FBGs was at half the distance between the rim and the bridge (Media 1, Media 2, Media 3). In order to compare the performance of the fiber cavity to a standard acoustic transducer, the reflection from the cavity was recorded on one channel of the USB interface while the other channel simultaneously recorded that of a preamplified high-quality piezoelectric (PZT) pickup built into the acoustic guitar (B-Band, A4). For the dynamic vibration measurements a calibrated accelerometer (Brüel \& Kjær, 4333) was used together with an amplifier of known gain (Brüel \& Kjær, 2635).

The FBG-FP cavities were used on a number of instruments aside from the guitar, with varying results. The sensor was attached to a clarinet (Media 4), a violin (Media 5, Media 6, Media 7), a solid body bass guitar (Media 8), and a harmonica

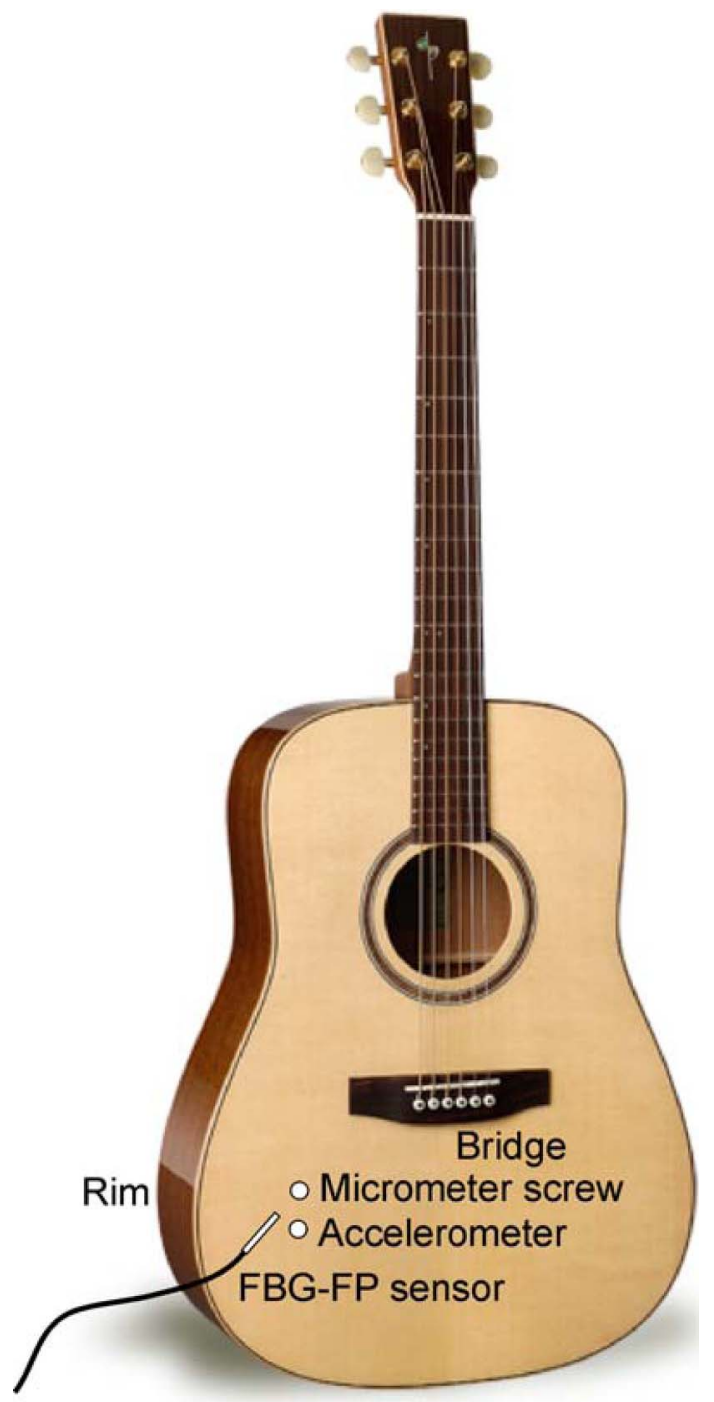

Fig. 2. (Color online) Photograph of the acoustic guitar (@2006 Godin Guitars). The approximate positions of the FBG-FP cavity sensor head and the accelerometer are indicated.
(Media 9) in order to test its response to vibrations of the instrument body.

\section{Results and Discussion}

\section{A. Strain and Acceleration Measurements}

The spectrum of the FBG-FP cavity is shown in Fig. 1(c) and is indicative of a cavity with low finesse $(<5)$. The free-spectral range of $10.1( \pm 0.3) \mathrm{GHz}$ together with the fiber core mode's refractive index of 1.4682 at $1550 \mathrm{~nm}$ indicates that the effective length of the cavity is $10.1 \mathrm{~mm}$ in excellent agreement with the manufacturer's specifications. The cavity length measured from the free-spectral range corresponds to the distance between the center of the two FBGs and needs to be corrected by the dimensions of the two FBGs $(300 \mu \mathrm{m})$ to obtain the physical length of the sensor element, here $10.4 \mathrm{~mm}$.

The strain response of the fiber cavity is determined in two ways. First, the fiber cavity was affixed to the soundboard of the guitar and the board was flexed at a point about $5 \mathrm{~cm}$ from the rim of the guitar body using a micrometer screw. The shift of the cavity spectrum then serves as a measure for the strain, and the strain response to vertical displacement of the soundboard can be determined. A second approach uses a calibrated accelerometer to correlate the amplitude of the FBG-FP cavity signal to the vertical displacement of the sound board. In all calculations we assume that the already low finesse of the cavity is not further reduced by the applied strain. This is supported by the spectra shown in Fig. 1(d), which show a shift but not a reduction in amplitude.

The wavelength shift of the FBG-FP cavity spectrum with increasing displacement on the soundboard [Fig. 1(d)] appeared linear with a slope of $-6.5( \pm 0.1) 10^{-5} \mathrm{~nm}$ per micrometer vertical displacement (Fig. 3). The strain on the cavity as a function of displacement is then readily calculated as $41 \mathrm{n} \varepsilon / \mu \mathrm{m}$. A simple model based on a one-dimensional elastic rod predicts strain of similar magnitude and a rod length of $43 \mathrm{~mm}$ (obtained by fitting) appears consistent to the distance between the sensor head and the rim of the guitar. However, this model does not reproduce the linearity of the increase of strain with the vertical displacement of one of the rod ends (see Fig. 3 inset). A more realistic model for the guitar body deformations should take into consideration that the restoring forces are larger near the rim of the guitar body, due to the curvature of the rim and to the braces that are placed inside the guitar to reinforce the construction. Details on both models are given in supplementary material [14]. To summarize the result, the deformations shown in the inset of Fig. 3 (curved black lines) reproduce the experimental strain values very well and were calculated assuming that the FBGFP cavity is placed along a circularly curved board. The radius of curvature is calculated by fitting as $7.9 \mathrm{~m}$, and the origin is calculated to be $2.0 \mathrm{~cm}$ to $6.0 \mathrm{~cm}$ from the point at which the load is applied (Fig. 3 inset). 


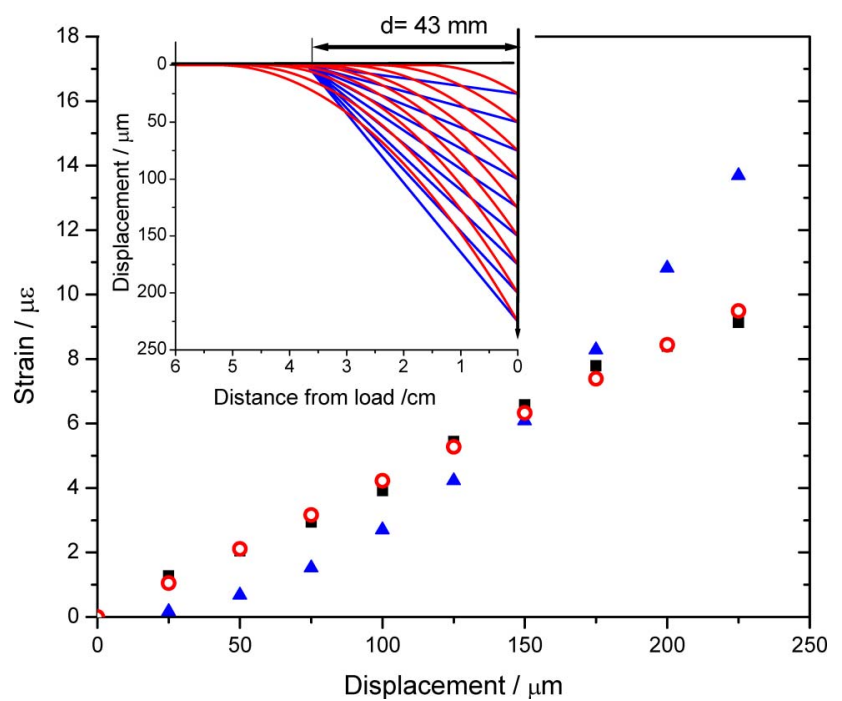

Fig. 3. (Color online) Strain on the FBG-FP cavity measured from the spectral shift in Fig. 1(d) (squares) and calculated from model 1 , in which the strain on the FBG-FP cavity is $\left(d^{2}+\right.$ $\left.1850 \mathrm{~cm}^{2}\right)^{1 / 2} / 4 \mathrm{~mm}-1$ (triangles). The empty circles are the result of model 2 , in which the bending radius is constant but the length of the deformed plate changes. The inset shows a cross section through the soundboard according to model 1 [straight (blue online) lines] and according to the more realistic model 2 [curved (red online) lines]; see text and supplementary material [14].

The dynamic response of the FBG-FP cavity sensor was also measured and calibrated against an accelerometer. We note that the FBG-FP cavity [Fig. 4(a)] is sensitive to both static and dynamic strain, whereas the PZT accelerometer is sensitive to acceleration only. The raw accelerometer data [Fig. 4(b)] were therefore twice numerically integrated to obtain a time series of vibration amplitude data [Fig. 4(c)]. The maximum amplitude of the soundboard for a plucked string (resonance frequency $300 \mathrm{~Hz}$ ) was found to be between 30 and $70 \mu \mathrm{m}$ depending on the force exerted by the guitar player, corresponding to a maximal FBG wavelength redshift of $2.0 \mathrm{pm}$ to $4.6 \mathrm{pm}$, and a strain on the FBG of $1.3 \mu \varepsilon$ to $2.9 \mu \varepsilon$, respectively. At these small wavelength shifts the intensity of the light reflected or transmitted through the FBG-FP cavity varies linearly with the vibration amplitude (Fig. 1). The light intensity at the wavelength of the midreflection point changes linearly by only $2.2 \%$ as the FBG-FP cavity spectrum is shifted by $1 \mathrm{pm}$. The linear range extends to wavelength shifts of up to $10 \mathrm{pm}$, corresponding to $6.5 \mu \varepsilon$. The maximum modulation of the light signal due to the plucked $\mathrm{E}_{2}$ string can be calculated from the sensitivity of the relative intensity change with vertical displacement $(0.15 \% / \mu \mathrm{m})$ and is about $4.4 \%$ to $10.1 \%$. Should a larger response (higher sensitivity) be desired, the cavity length will have to be extended or the reflectivity of the gratings needs to be increased. Of course, a cavity with higher finesse not only has increased sensitivity to strain but also a reduced range of vibration amplitude that may be measured in the linear response regime.

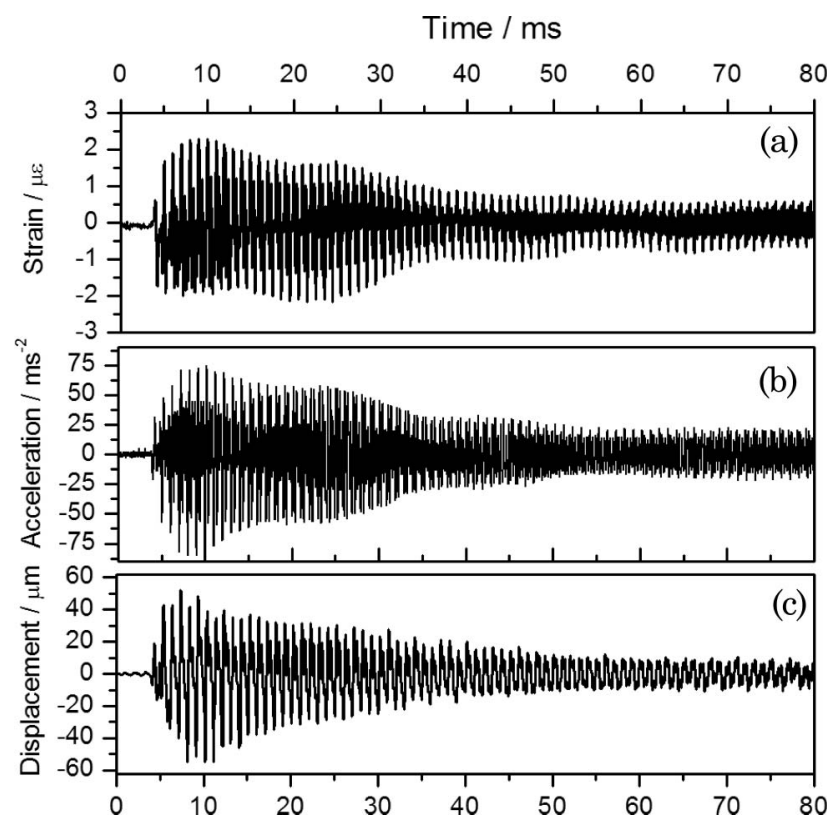

Fig. 4. Vibration of the soundboard measured with (a) the FBG-FP cavity and (b) an accelerometer. The raw data from the accelerometer were integrated twice to obtain the displacement amplitude (c). The strain on the FBG-FP cavity was calculated assuming the linear relation of Fig. $\underline{3}$.

\section{B. Audio Recordings}

While graphical representations of the FBG-FP cavity response are helpful to ascertain frequency range and linearity of the response, a direct comparison of audio recordings made with a high-quality PZT pickup and the FBG-FP cavity is more meaningful. To the journal's electronic database we have provided recordings of plucked strings and short melodies that were obtained using an acoustic guitar as described above (Media 1, Media 2, Media 3). Also, melodies and scales were recorded using a clarinet (Media 4), a violin (Media 5, Media 6, Media 7), and a harmonica (Media 8).

The audio recordings indicate a high recording quality, which approaches that of a conventional high-quality pickup. The main detriment to better performance lies in the sensitivity of the fiber-optic cables to movement. As demonstrated above, the audio signal is obtained from a light intensity modulation of at most $10 \%$. Light intensity fluctuations due to laser noise and cable losses may approach $1 \%$ and are then audible (Media 4, Media 9). In addition, birefringence induced by strain on the fiber cable causes intensity fluctuations at the polarizationsensitive fiber/fiber couplers. An added source of noise is due to the particular design of the laser driver. The device was designed to periodically reset the laser to the midreflection point of a cavity fringe. The process takes about $1 \mathrm{~ms}$ and produces an audible intensity spike (Media 1).

In an attempt to visualize the recording quality we show in Fig. 5 the FBG-FP cavity reflection recorded 


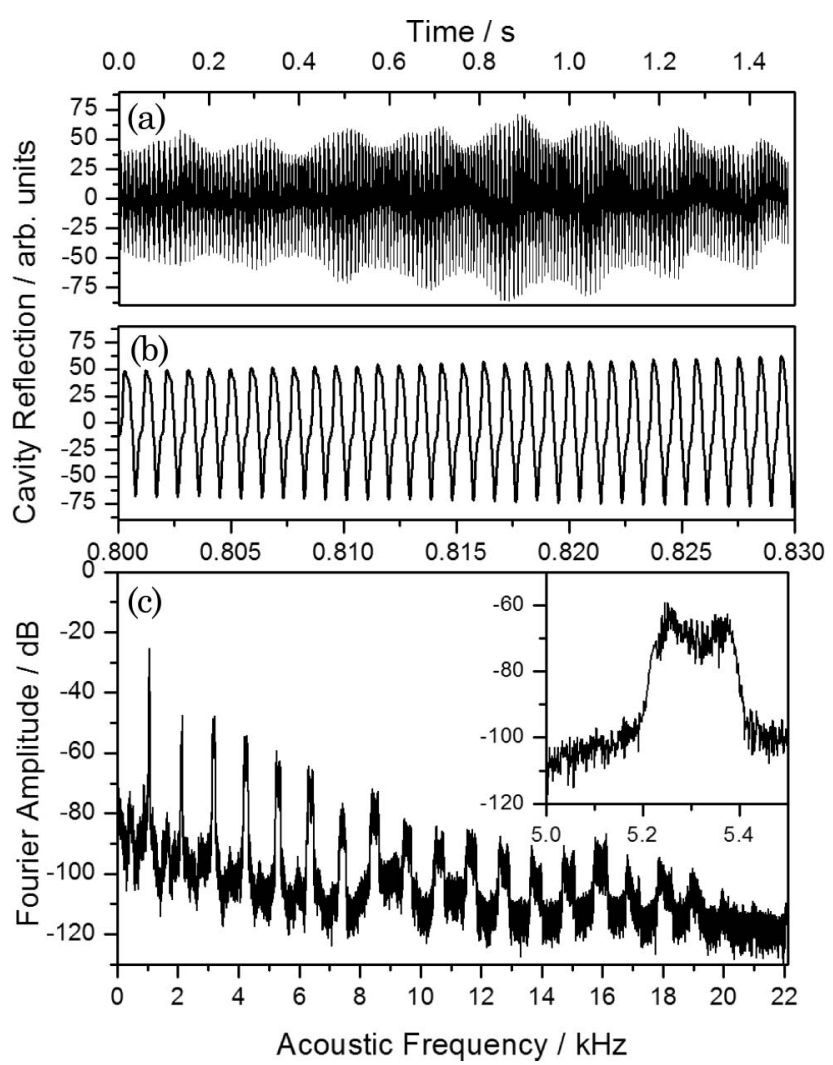

Fig. 5. (a) FBG reflection signal recorded when playing $\mathrm{C}_{6}$ $(1046.5 \mathrm{~Hz})$ on a violin. A $\sim 5 \mathrm{~Hz}$ vibrato is apparent as a modulation of the envelope (b) enlarged portion of the time trace (c) Fourier transform of the trace in panel (a). The split peaks (see the inset for the 5 th overtone) are also likely due to vibrato.

over $1.5 \mathrm{~s}$ when the cavity is attached to the body of a violin. The audio recording was part of a longer musical piece and was analyzed, here, without the attack on the string. The Fourier transform of this trace shows the fundamental frequency near $1050 \mathrm{~Hz}$ and harmonics up to the 18th harmonic at about $20 \mathrm{kHz}$, i.e., near the upper frequency limit that is audible by an adult.

\section{Conclusions}

With the present study we demonstrate that a simple FBG-FP cavity can serve as a transducer for audio frequency vibrations with amplitudes of a few micrometers. The finesse and free-spectral range of the fiber cavity were matched to the vibrations of the soundboard of a guitar. A musical instrument is a convenient system to test the performance of such a transducer, since the human ear is capable of recognizing sources of noise that are difficult to isolate otherwise. In the present case we were able to identify optical losses in the fiber-optic cables as the main limitation to a high-fidelity recording. Laser noise and noise arising from the laser driver design was noticeable but can be reduced by replacing mechanical fiber connectors with fusion splices. Also polarization control will reduce the noise due to the combined effect of birefringence in the fiber-optic cables and the polarization-sensitive coupling efficiency in the fiber couplers.

Compared to the previously described "photonic pickup," i.e. a single FBG, the present sensor was designed to have a lower sensitivity but larger linear dynamic range. The sensitivity is nevertheless still too large to reproduce the sound of "loud" instruments, such as a violin or even some acoustic guitars. When the vibration amplitude exceeds $200 \mu \mathrm{m}$ the spectrum shown in Fig. 1(d) shifts outside the linear response regime and the signal is audibly distorted.

While it is tempting to propose a commercial future for the "photonic guitar" or, more generally, for a fiber-optic based pickup, we note that two main obstacles need to be overcome: aside from the noise level which is higher than acceptable by high-fidelity audio standards, it is also necessary to reduce the cost of the system.

The authors acknowledge financial support from the Canadian Institute for Photonic Innovations, the Ontario Centres of Excellence, Queen's University, and PARTEQ Innovations. We are also grateful to Gianluca Gagliardi for many discussions on fiberoptic vibration sensing, and to Rui Resendes, Alex Kay, and Song Hee for recording the musical pieces. Finally, we thank luthiers Robert Godin (Godin Guitars) and Pete Swanson (Dagmar Guitars) for their advice on guitars and guitar pickups.

\section{References}

1. H. P. Loock, W. S. Hopkins, C. Morris-Blair, R. Resendes, J. Saari, and N. R. Trefiak, "Recording the sound of musical instruments with FBGs: the photonic pickup," Appl. Opt. 48, 2735-2741 (2009).

2. B. Sorazu, G. Thursby, B. Culshaw, F. Dong, S. G. Pierce, Y. Yang, and D. Betz, "Optical generation and detection of ultrasound," Strain 39, 111-114 (2003).

3. M. W. Hathaway, N. E. Fisher, D. J. Webb, C. N. Pannell, D. A. Jackson, L. R. Gavrilov, J. W. Hand, L. Zhang, and I. Bennion, "Combined ultrasound and temperature sensor using a fibre Brag grating," Opt. Commun. 171, 225-231 (1999).

4. N. E. Fisher, J. Surowiec, D. J. Webb, D. A. Jackson, L. R. Gavrilov, J. W. Hand, L. Zhang, and I. Bennion, "In-fibre Bragg gratings for ultrasonic medical applications," Meas. Sci. Technol. 8, 1050-1054 (1997).

5. N. Takahashi, K. Tetsumura, and S. Takahashi, "Underwater acoustic sensor using optical fiber Bragg grating as detecting element," Jpn. J. Appl. Phys. 38, 1 (1999).

6. N. Takahashi, K. Yoshimura, S. Takahashi, and K. Imamura, "Development of an optical fiber hydrophone with fiber Bragg grating," Ultrasonics 38, 581-585 (2000).

7. N. Takahashi, A. Hirose, and S. Takahashi, "Underwater acoustic sensor with fiber Bragg grating," Opt. Rev. 4, 691-694 (1997).

8. Q. X. Yang, J. Barnes, H. P. Loock, and D. Pedersen, "Time-resolved photoacoustic spectroscopy using fiber Bragg grating acoustic transducers," Opt. Commun. 276, 97-106 (2007).

9. Q. X. Yang, H. P. Loock, I. Kozin, and D. Pedersen, "Fiber Bragg grating photoacoustic detector for liquid chromatography," Analyst (Amsterdam) 133, 1567-1572 (2008). 
10. G. Gagliardi, M. Salza, P. Ferraro, and P. De Natale, "Interrogation of FBG-based strain sensors by means of laser radio-frequency modulation techniques," J. Opt. A Pure Appl. Opt. 8, S507-S513 (2006).

11. G. Gagliardi, M. Salza, P. Ferraro, and P. De Natale, "Fiber Bragg-grating strain sensor interrogation using laser radiofrequency modulation," Opt. Express 13, 2377-2384 (2005).
12. B. Lissak, A. Arie, and M. Tur, "Highly sensitive dynamic strain measurements by locking lasers to fiber Bragg gratings," Opt. Lett. 23, 1930-1932 (1998).

13. J. H. Chow, D. E. McClelland, M. B. Gray, and I. C. M. Littler, "Demonstration of a passive subpicostrain fiber strain sensor," Opt. Lett. 30, 1923-1925 (2005).

14. http://www.chem.queensu.ca/people/faculty/loock/. 